\title{
Histogram Equalization based Enhancement and MR Brain Image Skull Stripping using Mathematical Morphology
}

\author{
Zahid Ullah ${ }^{1}$, Prof. Su-Hyun Lee ${ }^{2} *$, Prof. Donghyeok $\mathrm{An}^{3}$ \\ Department of Computer Engineering \\ Changwon National University \\ Changwon, South Korea
}

\begin{abstract}
In brain image processing applications the skull stripping is an essential part to explore. In numerous medical image applications the skull stripping stage act as a preprocessing step as due to this stage the accuracy of diagnosis increases in the manifold. The MR image skull stripping stage removes the non-brain tissues from the brain part such as dura, skull, and scalp. Nowadays MRI is an emerging method for brain imaging. However, the existence of the skull region in the MR brain image and the low contrast are the two main drawbacks of magnetic resonance imaging. Therefore, we have proposed a method for contrast enhancement of brain MRI using histogram equalization techniques. While morphological image processing technique is used for skull stripping from MR brain image. We have implemented our proposed methodology in the MATLAB R2015a platform. Peak signal to noise ratio, Signal to noise ratio, Mean absolute error, Root mean square error has been used to evaluate the results of our presented method. The experimental results illustrate that our proposed method effectively enhance the image and remove the skull from the brain MRI.
\end{abstract}

Keywords-Contrast enhancement; skull stripping; magnetic resonance imaging; mathematical morphology

\section{INTRODUCTION}

Magnetic resonance imaging (MRI) is a non-invasive and an important imaging technique. MRI offers a high distinction of various soft tissues. Different applications of brain MRI, such as brain tissue segmentation, pathology detection, and multi-modal brain image registration need to extract the brain region as a preliminary step.

Medical brain imaging application is extensively used to detect different brain abnormalities like for instance paralysis, stroke, breathing difficulties and brain tumor. Skull stripping is an important pre-processing step in brain imaging since the last decade or so [1]. Segmentation is an important tool to study and diagnose various diseases such as autism [2], Alzheimer disease [3-5], and epilepsy [6] and it also required the recognition of anatomical structures, and brain tissue classification. The automatic skull stripping of a brain MRI is a challenging task due to low contrast, unclear brain boundaries, and pixels similarity. Using MRI datasets with a pathological disorder, the entire brain extraction becomes more challenging and problematic [37]. Nowadays, deep learning techniques such as convolution neural network-based algorithms, are mostly used to overcome medical imaging problems [38]. CNN based algorithm [39] employed known labeled data to learn the mathematical description required for the region or object detection, segmentation and classification.

The skull stripping removes skull, scalp, dura and skin/muscle from MR images for keeping only cerebral tissues. In MR image several brain diseases look similar specifically that disease which has an impact on cerebral atrophy. Those sensitive details cannot be differentiated by human naked eyes. Therefore, the enhancement of an image is necessary to accurately identify those details. The state-of-theart skull-stripping can be divided into four different classes, such as deformable surface model [7-10], thresholding with morphology [11, 12], region-based [13-15] and hybrid approaches [16-24].

The deformable surface model initially expresses the surface model and then repeatedly deforms the surface until it found the optimal solution. Brain extraction tool proposed by Smith [10], this method does not require any preprocessing/pre-registration before implementation. The region-based segmentation methodology takes the brain part as a single connected region. The region-based segmentation merges the same region into one larger region. This method comprised of watershed techniques [14], region growing $[13,15]$, etc. The hybrid technique integrated different existing methods to enhance performance.

The scan of the brain MRI consists of Axial, Coronal, and Sagittal view as shown in Fig. 1. We can obtain the Axial view by dividing the brain by a horizontal/ lateral plane. The Axial view divides the brain into two parts such as inferior and superior parts. However, the brain is divided into a Coronal view into ventral and dorsal parts by vertical/frontal plane. While, in the Sagittal view, the brain MR image is obtained by dividing the brain into right and left parts of the longitudinal/median plane. For different brain MR image applications, skull stripping is an important step like for instance brain strokes, brain tumor segmentation, Dyslexia, Epilepsy, and brain tissue segmentation. Also, the skull stripping method is used to remove the non-brain tissues such as skull, eyes, dura, scalp, etc. from MR brain images.

*Corresponding Author

E-mail: sleepl@changwon.ac.kr 


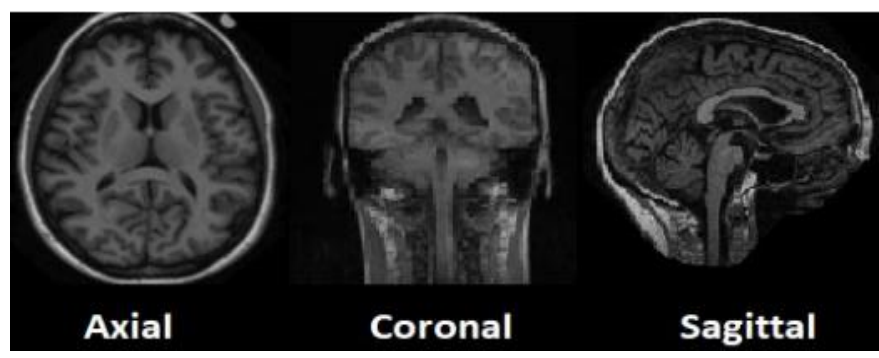

Fig. 1. Three Different views of the Brain.

In addition, there are numerous brain extraction algorithms, but the results of these algorithms on all brain MRI data sets are not satisfactory. Therefore, a robust and fully automated brain extraction algorithm is required which extracts the brain part accurately from a brain MRI database. MR brain volume exhibits numerous imaging artifacts, due to the limitations of material heterogeneity and spatial resolution of imaging modality, such as blurring, noise, partial volume effect, inhomogeneity and so on. Due to this imaging artifact, brain extraction becomes more difficult.

Considering all these limitations, we have proposed an accurate and robust skull stripping algorithm in this paper. We have used morphological based methods to remove the skull from the image while the histogram equalization based techniques have been used to enhance the brain MR image. As the magnetic resonance imaging modality generates the low contrast image and in this low contrast image it is very difficult for a doctor or radiologist to diagnose a disease. If the contrast of an image is high so the detail information can be easily analyzed. Therefore, we have enhanced every MR image as a pre-processing step using different histogram equalization techniques. In the human head, the skull is the hardest part and the skull act as a protector for the brain. However, diagnosing different brain diseases, the skull is a redundant part and it must be removed from the image. Therefore we have proposed an efficient methodology for skull stripping using mathematical morphology technique. In literature [25-28], several methods such as semi and fully automated methods are presented for skull removal.

\section{BRAIN MR IMAGE}

Magnetic resonance imaging is the most effective imaging modality to study the brains because the MRI has the capability to image the brain structures both interior and exterior with a high spatial resolution image of anatomical details, therefore, minute changes can be read or detected in these structures. The magnetic resonance imaging can generate images in any direction from side to side, top to bottom, or front to back. That's why the three-dimensional brain MR images are getting popular day by day in medical applications and also being used for research-related treatment, diagnosis, image-guided surgeries, and surgical planning.

The MR brain images are having three different types of images, T1-weighted, T2-weighted, and PD-weighted, where each type of MR brain image is focused on various contrast characteristics of the brain tissues [33]. The magnetic resonance brain imaging has got so many advantages as compared to other imaging modalities. The brain MR images are clearer and showing more detail related to other existing imaging modalities. MR imaging has the tendency to image the brain in any plane without moving the patient physically whereas computer tomography is limited to only one plane, which is an axial plane [34-35]. Due to these features, the MRI is an invaluable tool to evaluate or diagnose different brain diseases.

The MR brain imaging has been extensively used to diagnose different brain diseases, like Alzheimer disease, arteriogram, blood clots, brain tumor, Huntington's disease, hypopituitarism, stroke, multiple sclerosis, optic glioma, petit mal seizure, partial seizure, subdural hematoma, Cushing disease, etc. [36]. There are so many brain diseases, some of them are shown in Fig. 2.

The structure of the rest of the review paper is as follows: Section 3 presents the literature review, Section 4 presents the proposed model, Section 5 presents results and discussion, Section 6, presents Conclusion and Future Work.
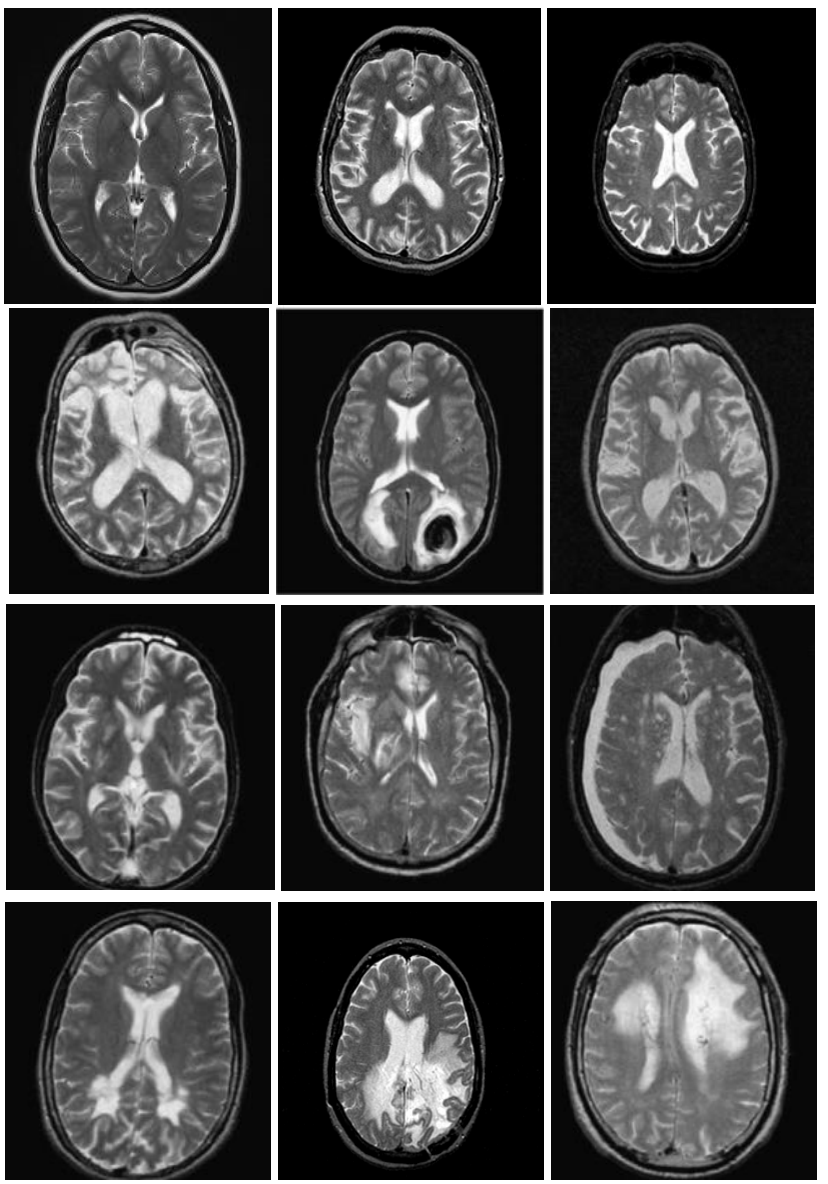

Fig. 2. Different brain MR images samples: a) Normal, b) Alzheimer's disease, c) Alzheimer's disease plus visual agnosia, d) Pick's disease, e) sarcoma, f) Huntington's disease, g) cerebral toxoplasmosis, h) herpes encephalitis, i) chronic subdural hematoma, j) multiple sclerosis, k) glioma, and 1) meningioma. 


\section{LITERATURE REVIEW}

Iglesias et al. [29] presented ROBEX (robust learningbased brain extraction tool). The generative and discriminative models are combined after standardizing signal intensities and bias filed correction is implemented. BEaST (Brain Extraction based segmentation) is another contemporary method [30]. Spatial and intensity normalization of the data is important in this method. The current methods are effective for some specific datasets but unfortunately not appropriate for others. Mathematical morphology proposed by Gonzales and woods [31] is an effective methodology for extracting skeleton, convex hull and other boundaries. For brain segmentation and analysis mathematical morphology has been used by different researchers $[7,11]$. They have used the morphological opening for brain tissue separation from the surrounding tissues while morphological closing and dilation have been employed to fill the holes in the image. As for further processing of an image, a binary form image is required for morphological operation. From the gray level image, the threshold creates a binary image by converting all the pixels values to zero which are below the threshold and those pixels which are above the threshold value are considered as one. However, the selection of a robust threshold value is a challenging task. In [16-24], hybrid approaches have been used for extracting the initial brain region, morphology-based method, in these cases, they have used the intensity thresholding. Further, the final binary brain mask is generated for various morphological tasks. The selection of accurate threshold value in these approaches is very challenging to find the region of interest. In reference [40], a survey can be seen on all the existing conventional skull-stripping methods. The state-of-art work can be seen in reference [41-47].

\section{Proposed Methodology}

MRI is the most effective imaging modality to study the brain tissues thoroughly as the MRI has the capability to capture the image structures both internally and externally with a high spatial resolution of anatomical details, therefore, minute changes can be read or detected in these structures. There are so many applications of brain imaging in the medical science field [7]. For these applications, the MR images are commonly used. In this paper, we have presented a robust algorithm for contrast enhancement and skull removal.

\section{CONTRAST ENHANCEMENT}

The MR imaging modality usually generates low-quality images and extracting information from a low-quality image is not an easy task. Therefore, in the first stage of our proposed methodology, we have presented an efficient technique for MRI contrast enhancement based on histogram equalization techniques such as:

\section{A. Median Filter}

The median filter is used in the pre-processing stage to the MR brain image for the removal of salt and pepper noise. As the MR image consists of salt and pepper and rician noises. The median filter removes the noises from MR images effectively while preserves the edges of the image efficiently. The median filter is a non-linear filter and this filter proceeds in such a way where it considers every pixel by the median value of the neighboring pixel. We have used a 3 x 3 window size for image filtering, as this window size is a suitable window size to filter an image.

\section{B. Histogram Equalization}

The HE can be represented as the mapping or transformation of every pixel of the input image into corresponding pixels of the processed output image [31]. The function of histogram equalization is to adjust the image intensities to improve the image contrast. The equation of histogram equalization is as follows:

$\mathrm{P}_{\mathrm{n}}=\frac{\text { no.of pixels with intensity } n_{k}}{\text { total no.of pixel } n}$

The range of the MR gray level image is $[0 \ldots \mathrm{L}-1]$.

\section{Adaptive Histogram Equalization}

In the fourth stage of the proposed methodology, we have used AHE, as this technique is effective for medical images to enhance the contrast of the image. Adaptive HE does not apply transformation or mapping on the overall image, but it performs separately on the sub-image and then combine the image in a proper way.

Pseudo-code of our proposed methodology is given below:

\begin{tabular}{l} 
Algorithm: Brain MRI Enhancement and Skull Stripping \\
\hline Input: brain MR image \\
Parameter: N is the total number of images \\
Step 1 (Median filter, HE, AHE, and CLAHE \\
For I = 1 : N \\
Read the images and implement the above techniques \\
//Enhanced Image \\
End \\
Step 2 Skull Stripping using mathematical morphology \\
For I = N \\
Read the enhanced brain MRI \\
Binarized the image using Otsu thresholding \\
Extract the largest connect component from the binary \\
image \\
End \\
Output: Skull Stripped Image
\end{tabular}

\section{Contrast Limited Adaptive Histogram Equalization}

CLAHE is an extension of the adaptive HE technique [36]. CLAHE and AHE are specifically used to curb the overenhancement problem of HE. CLAHE is employed to control the noise problem which is existed in traditional histogram equalization. In the MRI image, CLAHE works on the small regions which are known as tiles and it also calculates different histograms, and then compares each histogram to a specific part of the image and furthermore, it is utilized to reorganize the contrast estimation or brightness of the image. CLAHE provides more details as compare to standard histogram equalization as CLAHE improves the contrast of the image effectively but CLAHE still has the inclination to amplify unwanted pixels that have to be improved in the future work. The enhanced result of the gray level $l$ is computed by employing the below equation: 
$Y l=T(l)=\frac{R-1}{N} \sum_{k=o}^{l} H(k)$

Where $T(l)$ illustrates the mapping function and plots the different levels $l$ of the input picture into $y_{l}$.

\section{SKULL STRIPPING}

In MR brain imaging application the removal of the skull is a major stage and separation of non-cerebral tissues from cerebral tissues is known as skull stripping. In the skull removal process, the key problem is the separation of intracranial and non-cerebral tissues due to their intensities similarity. So we have presented an efficient methodology to overcome this issue by employing a mathematical morphology-based method as shown in Fig. 3.

\section{A. Otsu Thresholding}

The Otsu algorithm uses the zeroth and the first order cumulative moment of the gray level histogram. This algorithm is one of the simplest algorithms and is shown as follows:

$P_{i}=\frac{n_{i}}{N}, P_{i} \geq 0, \sum_{i=1}^{L} P_{i}=1$

\section{B. Mathematical Morphology and Hole Filling}

The morphological operations are implemented on a binary image such as erosion, dilation, and region filling to separate redundant areas. The binary image is convolved with a structuring element to generate the skull removal picture. As the structure of the brain is like an oval shape, therefore we consider a disk-shaped structuring element in the process of convolution as shown in Fig. 4.

We have used erosion to remove the pixel's which are residing on the boundaries of brain MR image and is also used for the elimination of non-brain regions such as meninges and skull. In reference [31] explains the erosion of a binary image as follows, $A$ employs structuring element while $B$ can be represented as follows:

$\mathrm{A} \Theta \mathrm{B}=\left\{\mathrm{Z} \mid(\mathrm{B})_{z} \subseteq \mathrm{A}\right\}$

The above equation can be explained as [31], erosion of $\mathrm{A}$ by $\mathrm{B}$ is the set of all points $\mathrm{z}$ such that $B$, translated by $\mathrm{z}$, is contained in $A$. While dilation can be defined as,

$$
\mathrm{A} \oplus \mathrm{B}=\left\{\mathrm{Z} \text { such that }(\hat{B})_{z} \cap \mathrm{A} \neq \varnothing\right\}
$$

The morphological dilation is employed in the image to unite entire intracranial tissues in the picture and can be explained as, dilation of A by B is the set of all displacements, $\mathrm{z}$, such that $\widehat{B}$ and $A$ overlap by at least one element.

Enhanced

Image

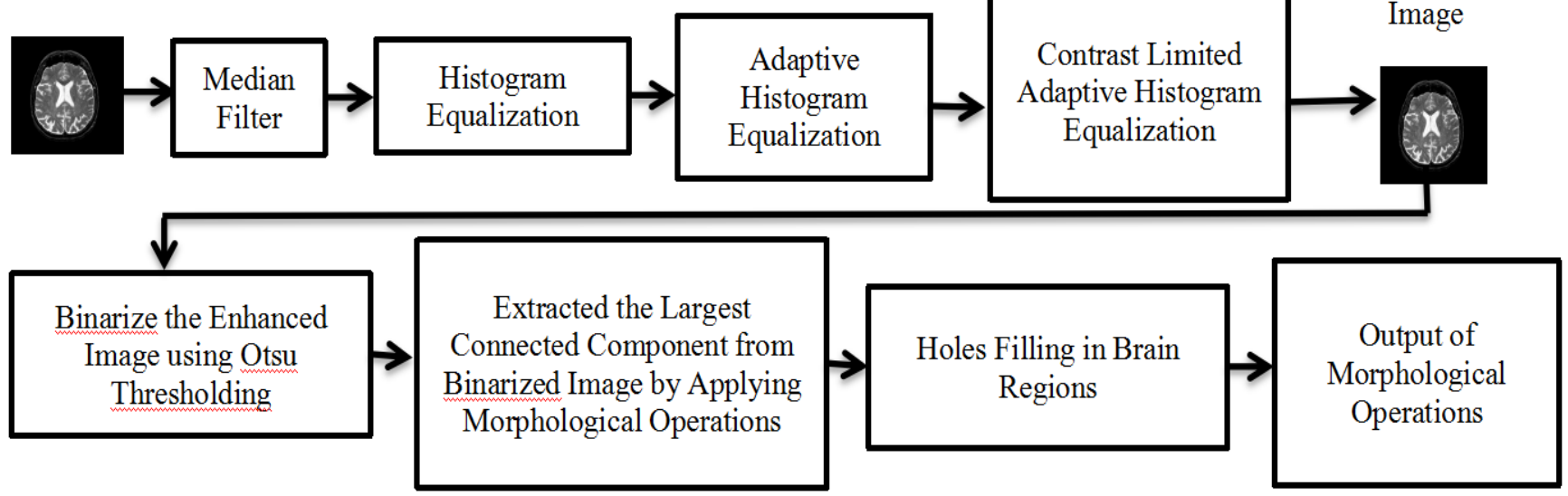

Fig. 3. Proposed Methodology for Brain Image enhancement and Skull Stripping.

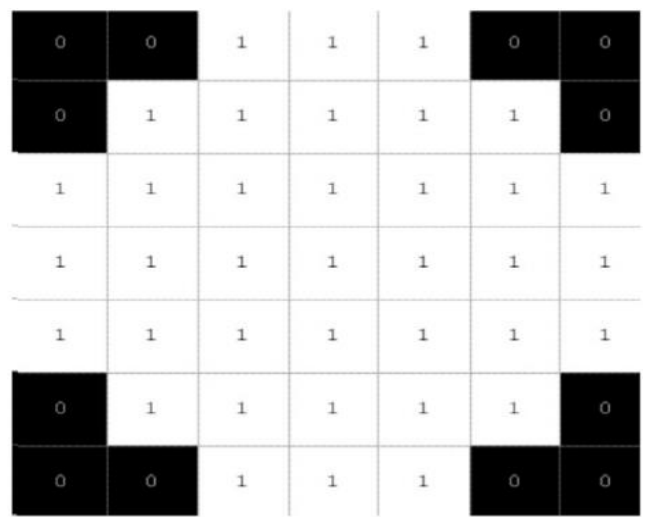

Fig. 4. Morphological Erosion and Dilation Structuring Element. 


\section{RESULTS AND DISCUSSION}

The simulation has been carried out using MATLAB 2015a to validate the proposed scheme on a personal computer with $3.30 \mathrm{GHz}$ Core-i5 processor and $4 \mathrm{~GB}$ RAM, running under Windows 10 operating system. Different types of medical image databases are being used for image segmentation. In this study, the database of brain MR images is collected from Harvard Medical School website, and the URL is http://www.med.harvard.edu/aanlib/home.html [32].

The magnetic resonance imaging modality produces a low contrast image. Therefore, in the proposed methodology, first, we have enhanced the brain MR image by using histogram equalization techniques as illustrated in Fig. 5. Secondly, we have considered this enhanced MR brain image for further processing of removal of the skull from the brain part by using mathematical morphology techniques as illustrated in Fig. 6. It has been noted that the results of our presented methodology can be comparable to other [26 and 27] morphological based skull stripping methods stated in the literature.

\section{A. Evaluation Metrics}

The efficiency of image contrast enhancement has been measured using Peak signal to noise ratio, Signal to noise ratio, Mean absolute error, Root mean square error [48]. The performance of image contrast enhancement is depicted in Table I. While the equations have been illustrated.

$$
\begin{aligned}
& P S N R=10 \log _{10}\left[\frac{\max (r(x, y))^{2}}{\frac{\sum_{0}^{n} x-1 \sum_{0}^{n} y-1[r(x, y)-t(x, y)]^{2}}{n x . n y}}\right] \\
& R M S E=\sqrt{\frac{\sum_{0}^{n} x-1 \sum_{0}^{n} y-1[r(x, y)-t(x, y)]^{2}}{n x . n y}} \\
& M A E=\frac{\sum_{0}^{n} x-1 \sum_{0}^{n} y-1|r(x, y)-t(x, y)|}{n x . n y}
\end{aligned}
$$

$$
S N R=10 . \log _{10}\left[\frac{\sum_{0}^{n} x-1 \sum_{0}^{n} y-1[r(x, y)]^{2}}{\sum_{0}^{n} x-1 \sum_{0}^{n} y-1[r(x, y)-t(x, y)]^{2}}\right]
$$

Fig. 5 to 9 illustrates different types of enhanced and respective skull stripping images using the proposed methodology.

In this study, the comparison between skull stripping and manually marked ground truth has been done using two standards such as Jaccard Coefficient and similarity coefficient Dice [49] as depicted in Table II. The proposed methodology has also been implemented on various MR brain image sequences as shown in Fig. 7 to 9.

JaccardCoefficient $=\frac{A(S) \cap A(G)}{A(S) \cup A(G)}$

DiceCoefficient $=\frac{2|A(S) \cap A(G)|}{|A(S)|+|A(G)|}$

It has been observed from the experimental results that the proposed methodology can be useful for different image analysis applications such as tumor classification, segmentation, and characterization.

TABLE. I. PERFORMANCE OF CONTRAST ENHANCEMENT ALgORITHM

\begin{tabular}{|l|}
\hline PSNR RMSE MAE SNR \\
\hline T2-W 25.1 12.65 .922 .9 \\
T1-W 26.6 11.45 .023 .3 \\
FLAIR 29.1 11.14 .923 .7 \\
DW1 27.4 10.74 .824 .5 \\
Avg. Perfor. 27.1 11.55 .023 .6 \\
\hline
\end{tabular}

TABLE. II. PERFORMANCE OF SKULl STRIPPING AlgORITHM

Dice Jaccard
T2-W 92.489 .9
T1-W 93.0 90.1
FLAIR 95.6 91.3
DW1 96.192 .1
Avg. Perfor. 94.390 .8

Dice Jaccard

Avg. Perfor. 94.390 .8 

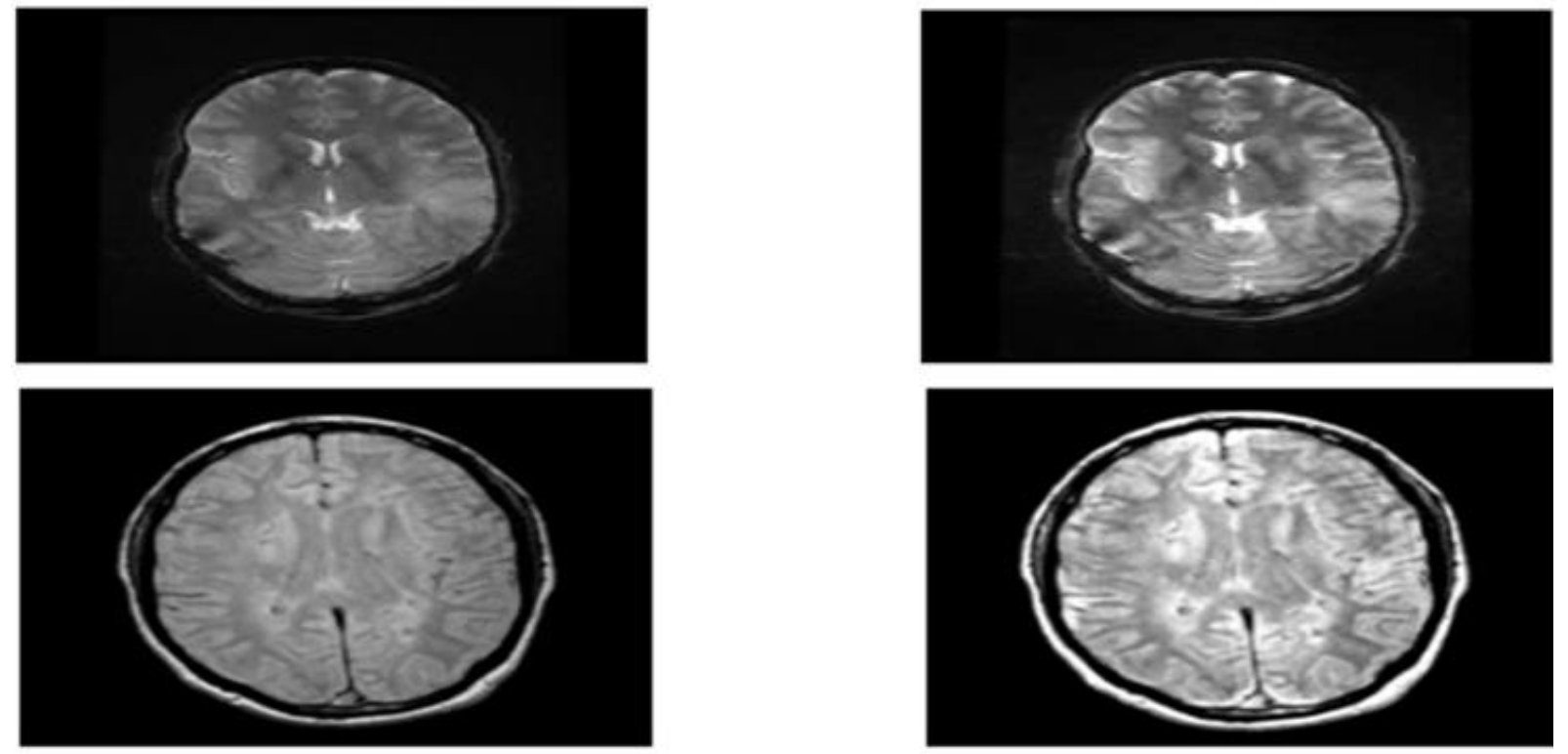

Fig. 5. Left Column Illustrates Original Input MR Brain Images while the Right Column Illustrates enhanced Output Images of MR Brain Images.
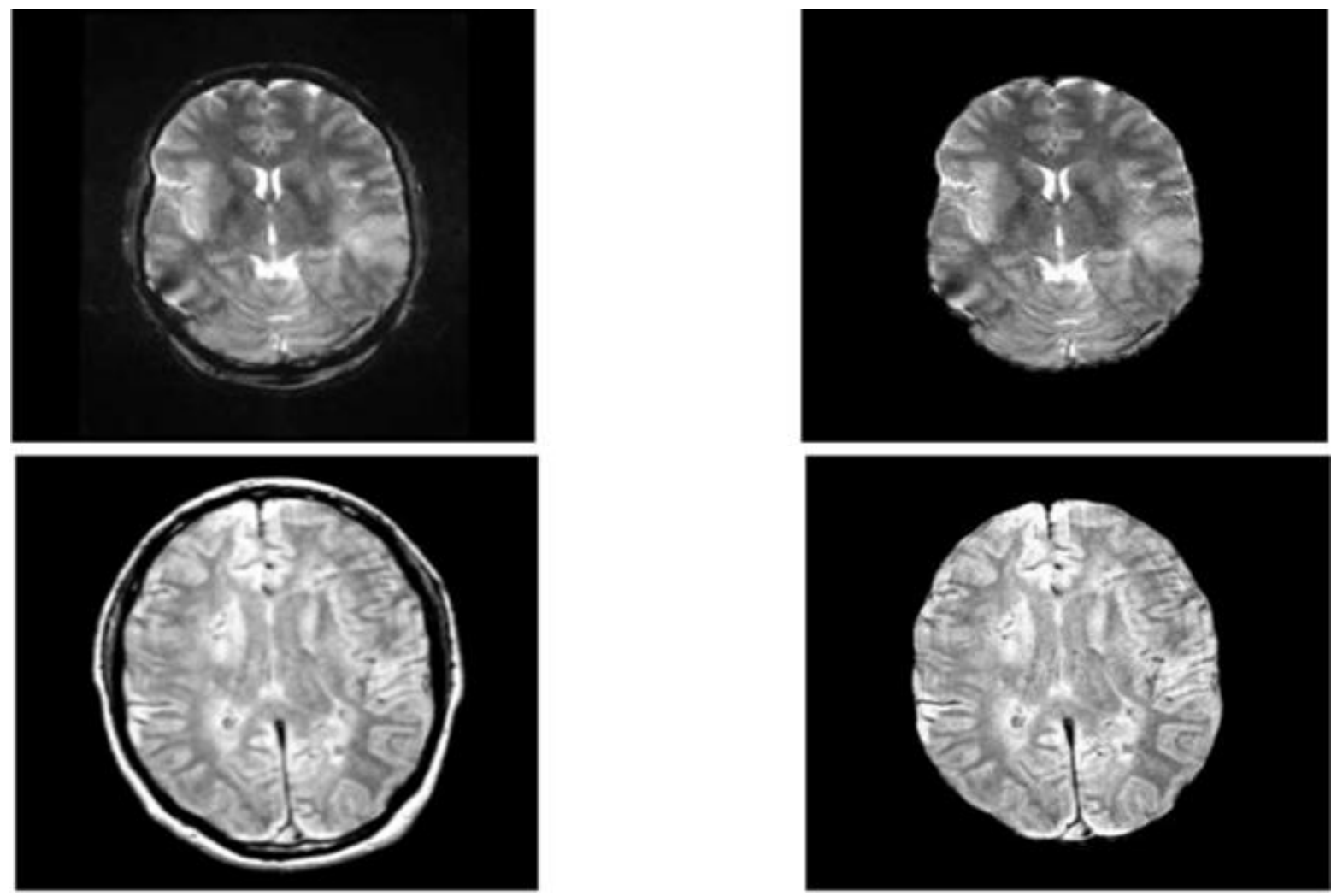

Fig. 6. Left Column Illustrates T2-W enhanced MR Images having Skull. While the Right Column Illustrates the Output of Skull Stripping Images. 

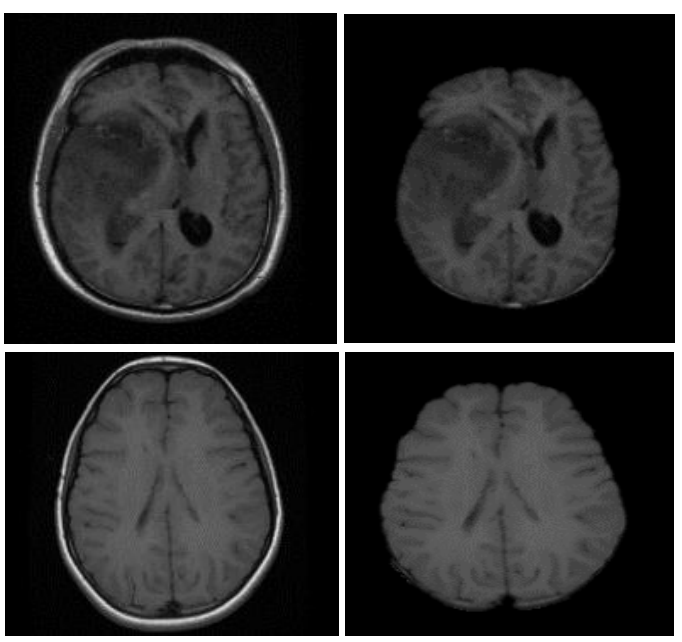

Fig. 7. Left Column Illustrates T1-W enhanced MR Images having Skull. While the Right Column Illustrates the Output of T1-W Skull Stripping Images.
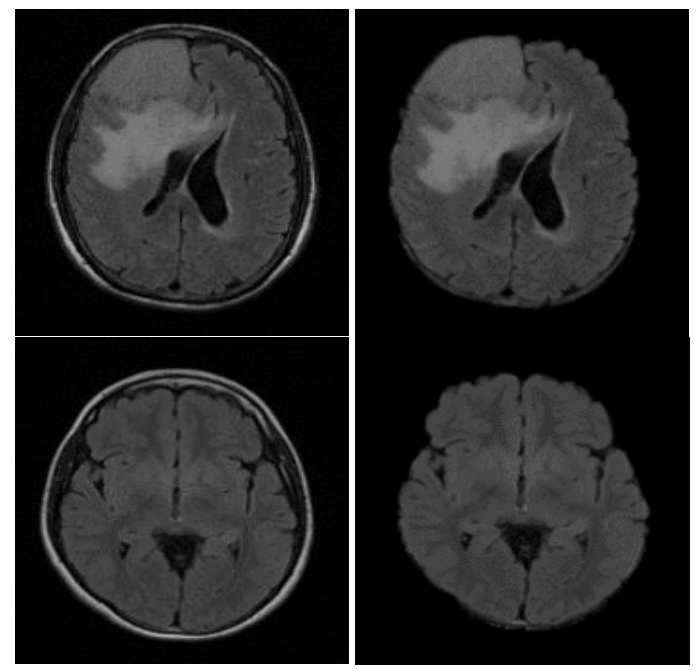

Fig. 8. Illustrates FLAIR enhanced Image and Respective Skull Stripped Image.
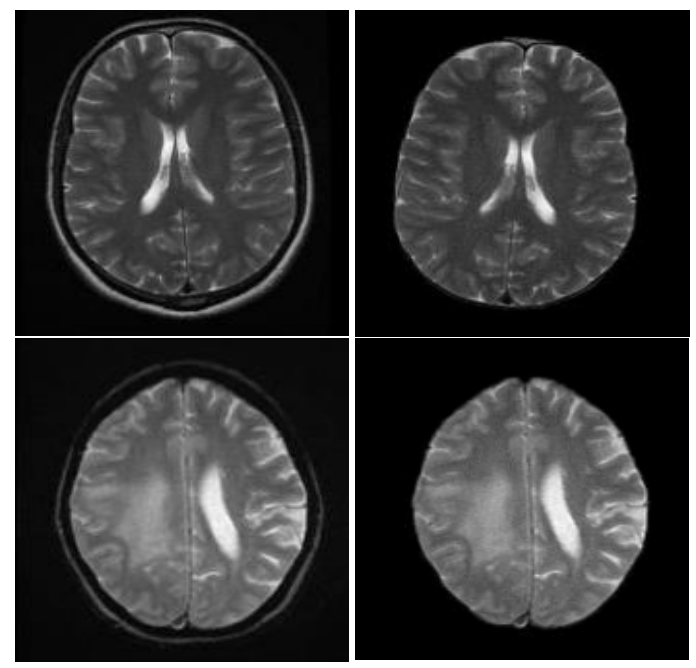

Fig. 9. Illustrates DW1 enhanced Image and Respective Skull Stripped Image.

\section{CONCLUSION}

In medical image segmentation applications, image preprocessing is an essential step to maximize classification accuracy. The images of MR imaging modality are low contrast and comprised of rician noise and salt and pepper noise. Therefore, these kinds of brain MR images are not helpful for physicians to diagnose a disease. To overcome this problem we have used histogram equalization techniques to enhance the brain MR images. However, the removal of the skull part from the brain part is also very helpful for the physicians to diagnose a disease accurately. So for skull stripping, we have used mathematical morphology techniques. This proposed algorithm works effectively on 2D MR brain images. It has been observed from the results that this methodology can be employed with many MR brain imaging applications and can be comparable to other morphological based skull stripping method.

\section{FUTURE WORK}

In future work, we will focus on the solution of similar intensity segmentation of the intracranial and non-cerebral tissue of the brain. Also, this proposed study can be evolved for the preprocessing of 3D MR brain images.

\section{CONFLICT OF INTEREST}

The authors declare that there is no conflict of interest regarding the publication of this paper.

\section{ACKNOWLEDGMENTS}

We are grateful to Department of Computer Engineering, Changwon National University for the immense support in piloting this work. This work was supported by the National Research Foundation of Korea (NRF) grant funded by the Korea government (MSIT) (No. 2018R1C1B6008187).

\section{REFERENCES}

[1] H. K. Hahn and H. O. Peitgen, "The skull stripping problem in MRI solved by a single 3D watershed transform," Lect. Notes Comput. Sci. (including Subser. Lect. Notes Artif. Intell. Lect. Notes Bioinformatics), vol. 1935, pp. 134-143, 2000.

[2] A. El-Baz, M. F. Casanova, G. Gimel'Farb, M. Mott, and A. E. Switwala, "A new image analysis approach for automatic classification of autistic brains," 2007 4th IEEE Int. Symp. Biomed. Imaging From Nano to Macro - Proc., pp. 352-355, 2007.

[3] C.R. Guttmann, F.A. Jolesz, R. Kikinis, R.J. Killiany, M.B. Moss, T. Sandor, and M.S. Albert, "White matter changes with normal aging," Neurology, vol. 50, issue-4, pp. 972-978, April, 1998.

[4] P. F. Buckley et al., "Three-dimensional magnetic resonance-based morphometrics and ventricular dysmorphology in schizophrenia," Biol. Psychiatry, vol. 45, no. 1, pp. 62-67, 1999.

[5] E. Jackson, P. Narayana, J. Wolinsky and T. Doyle, "Accuracy and reproducibility in volumetric analysis of multiple," Jou. Comput. Assisted Tomography, vol. 17, issue-2, pp. 200-205, March-April, 1993.

[6] K. Jafari-Khouzani, M.-R. Siadat, H. Soltanian-Zadeh, and K. Elisevich, "Texture analysis of hippocampus for epilepsy," Med. Imaging 2003 Physiol. Funct. Methods, Syst. Appl., vol. 5031, p. 279, 2003.

[7] M. Stella Atkins and B. T. Mackiewich, "Fully automatic segmentation of the brain in MRI," IEEE Trans. Med. Imaging, vol. 17, no. 1, pp. 98107, 1998.

[8] R. W. . H. Cox J.S., “ AFNI: Software for analysis and visualization of functional magnetic resonance neuroimages.," Comput. Biomed. Res., vol. 29 , no. 29 , pp. $162-173,1996$. 
[9] A. Kelemen, G. Székely, and G. Gerig, "Elastic model-based segmentation of 3-D neuroradiological data sets," IEEE Trans. Med. Imaging, vol. 18, no. 10, pp. 828-839, 1999.

[10] S. M. Smith, "Fast Robust Automated Brain Extraction," vol. 155, pp. 143-155, 2002.

[11] L. Lemieux, G. Hagemann, K. Krakow, and F. G. Woermann, "Fast, accurate, and reproducible automatic segmentation of the brain in T1weighted volume MRI data," Magn. Reson. Med., vol. 42, no. 1, pp. 127-135, 1999.

[12] P. Maji and S. Roy, "Rough-fuzzy clustering and unsupervised feature selection for wavelet based MR image segmentation," PLoS One, vol. 10, no. 4, pp. 1-30, 2015.

[13] R. Adams and L. Bischof, "Seeded Region Growing," IEEE Trans. Pattern Anal. Mach. Intell., vol. 16, no. 6, pp. 641-647, 1994.

[14] H. K. Hahn and H. O. Peitgen, "The skull stripping problem in MRI solved by a single 3D watershed transform," Lect. Notes Comput. Sci. (including Subser. Lect. Notes Artif. Intell. Lect. Notes Bioinformatics), vol. 1935, pp. 134-143, 2000.

[15] J. G. Park and C. Lee, "Skull stripping based on region growing for magnetic resonance brain images," Neuroimage, vol. 47, no. 4, pp. 1394-1407, 2009

[16] S. F. Eskildsen et al., "BEaST: Brain extraction based on nonlocal segmentation technique," Neuroimage, vol. 59, no. 3, pp. 2362-2373, 2012.

[17] F. J. Galdames, F. Jaillet, and C. A. Perez, "An accurate skull stripping method based on simplex meshes and histogram analysis for magnetic resonance images," J. Neurosci. Methods, vol. 206, no. 2, pp. 103-119, 2012.

[18] R. A. Heckemann et al., "Brain extraction using label propagation and group agreement: Pincram," PLoS One, vol. 10, no. 7, pp. 1-18, 2015.

[19] J. E. Iglesias, C. Y. Liu, P. M. Thompson, and Z. Tu, "Robust brain extraction across datasets and comparison with publicly available methods," IEEE Trans. Med. Imaging, vol. 30, no. 9, pp. 1617-1634, 2011.

[20] J. E. Iglesias, C. Y. Liu, P. M. Thompson, and Z. Tu, "Robust brain extraction across datasets and comparison with publicly available methods," IEEE Trans. Med. Imaging, vol. 30, no. 9, pp. 1617-1634, 2011.

[21] S. Roy, J. A. Butman, and D. L. Pham, "Robust skull stripping using multiple MR image contrasts insensitive to pathology," Neuroimage, vol. 146, no. November, pp. 132-147, 2017.

[22] S. A. Sadananthan, W. Zheng, M. W. L. Chee, and V. Zagorodnov, "Skull stripping using graph cuts," Neuroimage, vol. 49, no. 1, pp. 225239, 2010.

[23] F. Ségonne et al., "A hybrid approach to the skull stripping problem in MRI," Neuroimage, vol. 22, no. 3, pp. 1060-1075, 2004.

[24] D. W. Shattuck and R. M. Leahy, "Brainsuite: An automated cortical surface identification tool," Lect. Notes Comput. Sci. (including Subser. Lect. Notes Artif. Intell. Lect. Notes Bioinformatics), vol. 1935, pp. 5061, 2000.

[25] F. Ségonne et al., "A hybrid approach to the skull stripping problem in MRI," Neuroimage, vol. 22, no. 3, pp. 1060-1075, 2004.

[26] R. Roslan, N. Jamil, and R. Mahmud, "Skull stripping of MRI brain images using mathematical morphology," Proc. 2010 IEEE EMBS Conf. Biomed. Eng. Sci. IECBES 2010, no. December, pp. 26-31, 2010.

[27] S. Mohsin, S. Sajjad, Z. Malik, and A. H. Abdullah, "Efficient Way of Skull Stripping in MRI to Detect Brain Tumor by Applying Morphological Operations, after Detection of False Background," Int. J. Inf. Educ. Technol., no. July 2019, pp. 335-337, 2012.

[28] S. Roy, S. Nag, I. K. Maitra, P. Samir, and K. Bandyopadhyay, "Artefact Removal and Skull Elimination from MRI of Brain Image," no. June, 2014.
[29] J. E. Iglesias, C. Y. Liu, P. M. Thompson, and Z. Tu, "Robust brain extraction across datasets and comparison with publicly available methods," IEEE Trans. Med. Imaging, vol. 30, no. 9, pp. 1617-1634, 2011.

[30] S. F. Eskildsen et al., "BEaST: Brain extraction based on nonlocal segmentation technique," Neuroimage, vol. 59, no. 3, pp. 2362-2373, 2012.

[31] R. C. Gonzales and R. E. Woods, Digital Image Processing, Second Edition, Prentice Hall, 2002.

[32] "Harvard Medical School Data," www.med.harvard.edu/AANLIB

[33] R. M. Quencer and W. G. Bradley, "MR imaging of the brain: What constitutes the minimum acceptable capability?," Am. J. Neuroradiol., vol. 22, no. 8, pp. 1449-1450, 2001.

[34] M. Cheour, Advantages of brain MRI, 2010, Available at, RadiologyInfo.org.

[35] P. Schmid, "Segmentation of digitized dermatoscopic images by twodimensional color clustering," IEEE Trans. Med. Imaging, vol. 18, no. 2, pp. 164-171, 1999.

[36] NLM-National Library of Medicine, (Rockville Pike, Bethesda U.S., 2011), Available online at: http://www.nlm.nih.gov.

[37] J. Kleesiek et al., "Deep MRI brain extraction: A 3D convolutional neural network for skull stripping," Neuroimage, vol. 129, pp. 460-469, 2016.

[38] G. Litjens et al., "A survey on deep learning in medical image analysis," Med. Image Anal., vol. 42, no. December 2012, pp. 60-88, 2017.

[39] Y. Lecun, Y. Bengio, and G. Hinton, “Deep learning," Nature, vol. 521, no. 7553, pp. 436-444, 2015.

[40] P. Kalavathi and V. B. S. Prasath, "Methods on Skull Stripping of MRI Head Scan Images - a Review," J. Digit. Imaging, vol. 29, no. 3, pp. 365-379, 2016.

[41] S. S. Mohseni Salehi, D. Erdogmus, and A. Gholipour, "Auto-Context Convolutional Neural Network (Auto-Net) for Brain Extraction in Magnetic Resonance Imaging," IEEE Trans. Med. Imaging, vol. 36, no. 11, pp. 2319-2330, 2017.

[42] N. H. M. Duy, N. M. Duy, M. T. N. Truong, P. T. Bao, and N. T. Binh, "Accurate brain extraction using Active Shape Model and Convolutional Neural Networks," no. February, 2018.

[43] R. Dey, Y. Hong, "CompNet: Complementary Segmentation Network for Brain MRI Extraction," arXiv 2018, preprint arXiv:1804.00521.

[44] Ö. Çiçek, A. Abdulkadir, S. S. Lienkamp, T. Brox, and O. Ronneberger, "3D U-net: Learning dense volumetric segmentation from sparse annotation," Lect. Notes Comput. Sci. (including Subser. Lect. Notes Artif. Intell. Lect. Notes Bioinformatics), vol. 9901 LNCS, no. June 2016, pp. 424-432, 2016.

[45] B. Puccio, J. P. Pooley, J. S. Pellman, E. C. Taverna, and R. C. Craddock, "The preprocessed connectomes project repository of manually corrected skull-stripped t1-weighted anatomical mri data," Gigascience, vol. 5, no. 1, pp. 1-7, 2016.

[46] Fischl, B. FreeSurfer. Neuroimage 2012, 62, 774-781, doi:10.1016/j.neuroimage.2012.01.021.

[47] A. Fedorov, J. Johnson, E. Damaraju, A. Ozerin, V. Calhoun, and S. Plis, "End-to-end learning of brain tissue segmentation from imperfect labeling," Proc. Int. Jt. Conf. Neural Networks, vol. 2017-May, pp. 3785-3792, 2017.

[48] K. J. Jang et al. "Measuremnet of Image Quality in CT Images Reconstructed with Different Kernels," Jour. Of the Korean Phy. Soc, 58(2), pp. 334-342, 2011.

[49] Jaccard, P.: The Distribution of Flora in Alpine Zone, New Phytol, $11(2), 1912,37-50$. 
AUTHOR's PROFILE

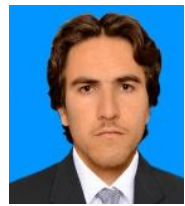

https://orcid.org/0000-0002-0184-7620

Zahid Ullah, is currently doing Ph.D in Computer Engineering from Changwon National University South Korea. He received MS in Computer Science from SZABIST, Islamabad, Pakistan in 2015, and BS in Information Technology from University of Malakand Pakistan in 2011. His area of interest are Image Processing, Medical Imaging, Computer Vision, and Machine Learning Techniques.

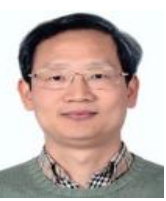

$$
\text { https//orcid.org/0000-0001-6966-1569 }
$$

Dr. Su-Hyun, Lee, is working as a Professor and Head of department of Computer Engineering, at Changwon National University South Korea. He is working in multiple desciplines, especially Bio-Informatics, Algorithm, and Programming.

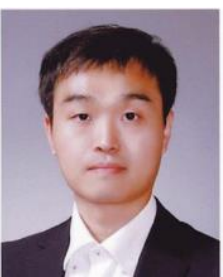

Dr. Donghyeok An received the B.S. degree in computer science from Handong University, Pohang, South Korea, in 2006, and the Ph.D. degree in Computer Science from Korea Advanced Institute of Science and Technology (KAIST) in Feb. 2013. He was a post doctor and a visiting professor at Sungkyunkwan University from Mar. 2013 to Feb. 2014. He was a senior engineer at Samsung Electronics from 2014 to 2015 . He was an assistant professor in the Department of Computer Engineering, Keimyung University from 2015 to 2017. He is currently an assistant professor in the Department of Computer Engineering, Changwon National University, Changwon, South Korea. His research interests include $5 \mathrm{G}$ networks, ultra reliable low latency communication, and Internet of Things. 